\title{
Liraglutide protects palmitate-induced INS-1 cell injury by enhancing autophagy mediated via FoxO1
}

\author{
XIU-DAN LI, SHAN-SHAN HE, TING-TING WAN and YAN-BO LI \\ Department of Endocrinology, First Affiliated Hospital of Harbin Medical University, \\ Harbin, Heilongjiang 150001, P.R. China
}

Received July 7, 2020; Accepted November 12, 2020

DOI: $10.3892 / \mathrm{mmr} .2020 .11786$

\begin{abstract}
Type 2 diabetes mellitus (T2DM) is characterized by insulin resistance and a progressive loss in mass and function of pancreatic $\beta$-cells. In T2DM, lipotoxicity leads to $\beta$-cells dysfunction and decreases its number. Autophagy serves a crucial role in maintaining the normal islet architecture and the function of $\beta$-cells. Moreover, glucagon-like peptide-1 (GLP-1) and its analogs have beneficial roles in pancreatic $\beta$-cells. However, the protective effects of GLP-1 agents on palmitate (PA)-induced pancreatic $\beta$-cells and their underlying mechanisms are not fully elucidated. Forkhead box $\mathrm{O} 1$ (FoxO1) can prevent pancreatic $\beta$-cells from apoptosis. Whether GLP-1 protects against PA-induced $\beta$-cells injury via FoxO1 remains unknown. The present study exposed INS-1 cells to PA to establish a T2DM injury model. Cell viability was evaluated using a Cell Counting Kit-8 assay, and apoptosis was determined via western blotting. Furthermore, autophagy was examined using western blotting, immunofluorescence and transmission electron microscopy. Silencing FoxO1 was used to inhibit the activities of FoxO1. The results suggested that the GLP-1 analog liraglutide enhanced the cell viability, inhibited the protein expression of cleaved caspase- 3 and increased the expression levels of microtubule-associated protein 1 light chain3 (LC3) II/I, and FoxO1 in INS-1 cells. The autophagy inhibitor chloroquine inhibited the protective effects of liraglutide on INS-1 cells. Silencing of FoxO1
\end{abstract}

Correspondence to: Professor Yan-Bo Li, Department of Endocrinology, First Affiliated Hospital of Harbin Medical University, 23 You Zheng Street, Harbin, Heilongjiang 150001, P.R. China

E-mail: liyanbo65@163.com

Abbreviations: CCK-8, Cell Counting Kit-8; ERs, endoplasmic reticulum stress; FFA, free fatty acids; FoxO1, Forkhead box O1; GLP-1, glucagon-like peptide-1; LC3, microtubule-associated protein 1 light chain3; p-FoxO1, phosphorylated FoxO1; siRNA, small interfering RNA; TEM, transmission electron microscopy; T2DM, type 2 diabetes mellitus

Key words: T2DM, pancreatic $\beta$ cells, autophagy, liraglutide, FoxO1 decreased the expression levels of LC3-II and attenuated the protection of liraglutide on the viability of INS-1 cells. In conclusion, the results indicated that liraglutide ameliorated the PA-induced islet $\beta$-cells injury via the upregulation of autophagy-mediated by FoxO1.

\section{Introduction}

Type 2 diabetes mellitus (T2DM) is characterized by insulin resistance and dysfunction of pancreatic $\beta$-cells. Enhanced pancreatic $\beta$-cells apoptosis causes $\beta$-cells loss in T2DM $(1,2)$. Therefore, the regulation of pancreatic $\beta$-cells mass and its function serve a critical role in the pathogenesis of T2DM. Currently, there are a limited number of drugs, such as liraglutide and metformin, approved for the prevention of islet $\beta$-cells from injury (1,3-5).

Autophagy is characterized by the lysosomal degradation of cellular material, and is a process of subcellular membrane rearrangement sequestering cytoplasm, proteins and organelles, forming the autophagosome (6). Previous studies have revealed that autophagy can prevent $\beta$-cells injury and death by protecting against endoplasmic reticulum stress (ERs), inflammation and oxidative stress (7-11). In our previous studies, autophagy was found to exert a protective effect by mitigating inflammation in $\beta$-cells $(1,8,10)$. It has also been reported that Atg7-KO ob/ob mice develop severe diabetes and have increased levels of apoptotic $\beta$-cells and decreased $\beta$-cell viability by imposing ERs (12). These studies revealed that autophagy serves a protective role in pancreatic $\beta$-cells.

Forkhead box O1 (FoxO1) belongs to the forkhead box protein family and is involved in a series of intracellular functions, including apoptosis, oxidative stress and mitochondrial dysfunction (13-16). FoxO1 is highly expressed in islet $\beta$-cells and serves a protective role in T2DM $(14,17)$. For instance, FoxO1 protects against $\beta$-cells failure, contributes to localize in the nucleus of $\beta$-cells and induces neuronal differentiation 1 and MAF bZIP transcription factor $\mathrm{A}$, which directly bind to the insulin 2 gene promoter and promotes its transcription (17). Furthermore, FoxO1 protects lipopolysaccharide-induced INS-1 cells from oxidative stress damage and mitochondrial dysfunction (16). In db/db mouse model, FoxO1 knockout decreases glucose-responsive insulin secretion (18). Moreover, FoxO1 regulates autophagic flux in various cell types, such as vascular endothelial cells (19), human cholangiocarcinoma 
QBC939 cells (15) and rat mesangial cells (20). However, the effect of FoxO1 on autophagy in pancreatic $\beta$-cell has not been fully elucidated.

Liraglutide is a glucagon-like peptide-1 (GLP-1) analog, which is known to regulate the synthesis of insulin, inhibits the apoptosis of pancreatic $\beta$-cells and is widely used for the treatment of T2DM. Liraglutide prevents high glucose or free fatty acids (FFA)-induced INS-1 cell apoptosis by targeting autophagy $(1,3)$. Fan et al (4) reported that liraglutide can enhance autophagy and promote islet $\beta$-cells proliferation in a high-fat-fed and streptozotocin-induced mouse model of T2DM. Moreover, liraglutide markedly improves islet $\beta$-cells function under lipid stress and blocks the inhibitory effect of palmitate (PA) on FoxO1 (21). It has also been shown that GLP-1 increases the levels of phosphorylated FoxO1 in $\beta$-cells (22). However, the molecular mechanism via which liraglutide exerts its action on autophagy and FoxO1 remains unknown.

Based on the autophagy functions and the roles of FoxO1 in the regulation of islet $\beta$-cells, it was hypothesized that the protective effect and corresponding mechanisms of liraglutide on pancreatic $\beta$-cells may be associated with induced autophagy and exerted by activating FoxO1. In the present study, INS-1 cells (induced by PA) were used to validate this hypothesis.

\section{Materials and methods}

Reagents and chemicals. INS-1 rat insulinoma cells were purchased from the American Type Culture Collection. RPMI-1640 medium was purchased from Thermo Fisher Scientific, Inc. FBS was purchased from Hangzhou Sijiqing Biological Engineering Materials Co. Ltd. Liraglutide was purchased from Novo Nordisk Hellas Ltd. PA and chloroquine (CQ) were obtained from Sigma-Aldrich (Merck KGaA). FoxO1 small interfering RNA (siRNA) was obtained from Shanghai GenePharma Co., Ltd. The following antibodies were used at a 1:1,000 dilution: Microtubule-associated protein 1 light chain3 (LC3; cat. no. 2775; Cell Signaling Technology, Inc.), phosphorylated (p)-FoxO1 (cat. no. 9461; Cell Signaling Technology, Inc.), FoxO1 (cat. no. 2880; Cell Signaling Technology, Inc.), cleaved caspase-3 (cat. no. AF1150; Beyotime Institute of Biotechnology), $\beta$-actin (cat. no. 8457; Cell Signaling Technology, Inc.) and GAPDH (cat. no. sc-32233; Santa Cruz Biotechnology, Inc.). Horseradish peroxidase-conjugated secondary antibodies were used at a 1:5,000 dilution, which included goat anti-rabbit and goat antimouse secondary antibodies from Jackson ImmunoResearch Laboratories, Inc. (cat. nos. 111-545-003 and 115-005-003). Cell Counting Kit-8 (CCK-8) was obtained from Dojindo Molecular Technologies, Inc. SDS-PAGE and an ECL detection kit were obtained from Cytiva.

Cell culturing. INS-1 cells were cultured in RPMI-1640 medium supplemented with $10 \%$ (v/v) FBS, in a humidified atmosphere containing $95 \%$ air and $5 \% \mathrm{CO}_{2}$ (1). All experiments were performed at $80 \%$ cell confluence. A PA-induced lipid toxicity model of INS-1 cells was established to investigate whether GLP-1 protects against PA-induced $\beta$-cells injury via FoxO1. CQ, a lysosome inhibitor, was used to block the autophagic flux (23). The concentration of CQ was selected according to a previous study (23). The concentration of liraglutide was chosen based on a previous study $(1,23)$.

First, purified INS-1 cells were incubated at $37^{\circ} \mathrm{C}$ and in various concentrations of PA $(0,0.1,0.3$ and $0.5 \mathrm{mmol} / \mathrm{l})$ for $24 \mathrm{~h}$. Then, INS-1 cells were divided into eight groups: i) Control (CON) group; ii) PA group (0.5 mmol/l); iii) $\mathrm{CQ}$ group $(10 \mu \mathrm{mol} / \mathrm{l})(22)$; iv) PA + CQ group (PA $0.5 \mathrm{mmol} / \mathrm{l}+$ CQ $10 \mu \mathrm{mol} / \mathrm{l})$; v) LIRA group (liraglutide $100 \mathrm{nmol} / \mathrm{l})(1,20)$; vi) PA + LIRA group (PA $0.5 \mathrm{mmol} / 1$ + liraglutide $100 \mathrm{nmol} / \mathrm{l}$ ); vii) LIRA + CQ group (liraglutide $100 \mathrm{nmol} / \mathrm{l}+\mathrm{CQ} 10 \mu \mathrm{mol} / \mathrm{l}$ ); and viii) $\mathrm{PA}+\mathrm{LIRA}+\mathrm{CQ}$ group (PA $0.5 \mathrm{mmol} / \mathrm{l}+$ liraglutide $100 \mathrm{nmol} / 1+\mathrm{CQ} 10 \mu \mathrm{mol} / \mathrm{l})$.

siRNA interference technology was used to inhibit FoxO1 gene expression in INS-1 cells. After the transfection, cells were randomly divided into four groups: PA + siCON group (PA $0.5 \mathrm{mmol} / 1+$ negative controls siRNA), $\mathrm{PA}+$ siFoxO1 group (PA $0.5 \mathrm{mmol} / 1+$ siFoxO1), LIRA + siCON group (liraglutide $100 \mathrm{nmol} / 1$ + negative controls siRNA) and LIRA + siFoxO1 group (liraglutide $100 \mathrm{nmol} / \mathrm{l}+\mathrm{siFoxO1}$ ).

Cell viability assay. Cell viability was assessed with a CCK-8 according to the manufacturer's protocol. INS-1 cells were treated with $10 \mu \mathrm{l} \mathrm{CCK}-8$ for $1 \mathrm{~h}$ at $37^{\circ} \mathrm{C}$. Absorbance was measured at $450 \mathrm{~nm}$ using a microplate reader (Tecan Group, Ltd.).

siRNA transfection. INS-1 cells were seeded in 6-well plates at a density of $2 \times 10^{5}$ per well with RPMI-1640 supplemented with $10 \% \mathrm{FBS}$ at $37^{\circ} \mathrm{C}$ for $24 \mathrm{~h}$. INS-1 cells were transfected with $80 \mathrm{nM}$ siRNA against FoxO1 or their negative controls duplexes using PepMute siRNA. The sequences of the designed siFoxO1 were as follows: FoxO1 siRNA, 5'-GGACAGCAAAUCAAGUUAUtt-3'. The PepMute ${ }^{\mathrm{TM}}$ siRNA transfection reagent (Signa Gen, SL100566) was used according to the manufacturer's instructions. INS-1 cells were transfected at $37^{\circ} \mathrm{C}$ for $24 \mathrm{~h}$, then treated with $0.5 \mathrm{mmol} / 1 \mathrm{PA}$ or $100 \mathrm{nmol} / 1$ liraglutide at $37^{\circ} \mathrm{C}$. After $24 \mathrm{~h}$ of treatment, protein extracts from cells were collected and used for western blot analysis.

Western blotting. INS-1 cells were harvested for $24 \mathrm{~h}$ as aforementioned. RIPA lysis buffer (cat. no. P0013K; Beyotime Institute of Biotechnology) was used to extract protein. Lysates were centrifuged at $4^{\circ} \mathrm{C}$ and $12,000 \mathrm{x} \mathrm{g}$ for $5 \mathrm{~min}$ and supernatant was collected. After using BCA protein assay kit (cat. no. P0012S; Beyotime Institute of Biotechnology) to determine the protein concentration, $45 \mu \mathrm{g}$ protein was boiled for $5 \mathrm{~min}$ and separated on 8 or $12 \%$ SDS-PAGE. The proteins were transferred to PVDF membrane, then $5 \%$ non-fat milk was used for blocking for $1 \mathrm{~h}$ at room temperature. After washing, the membranes were incubated with the primary antibody at $4{ }^{\circ} \mathrm{C}$ overnight. After washing, the membranes were incubated with the second antibody for $1 \mathrm{~h}$ at room temperature. Western blot analyses were performed using previously described methods $(1,16)$. The relative expression level of a specific protein was normalized to GAPDH. Immunoblots were semi-quantified via densitometric analysis using Image Lab software v2.0.1 (Bio-Rad Laboratories, Inc.).

Immunofluorescence assay. Following the aforementioned treatments, INS-1 cells were fixed at room temperature for 
A

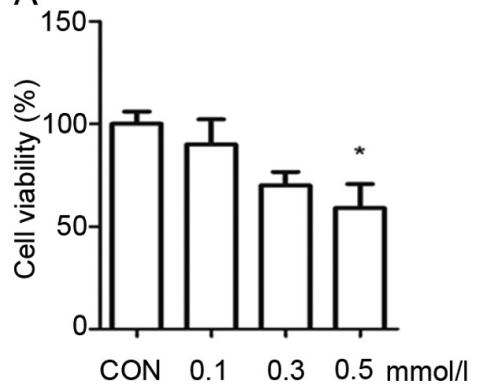

B

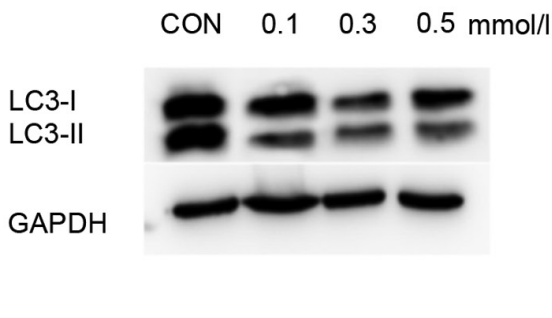

C

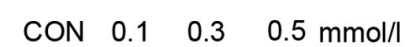

Figure 1. Effects of palmitate in INS-1 cells injury, autophagy and FoxO1. (A) Cell Counting Kit-8 assay was used to examine the viability of INS-1 cells, which were treated with various concentrations of palmitate $(0,0.1,0.3$ and $0.5 \mathrm{mmol} / \mathrm{l})$. Data were analyzed by unpaired $\mathrm{t}-\mathrm{test}$ and are presented as the mean $\pm \mathrm{SD}$ $(\mathrm{n}=3) .{ }^{*} \mathrm{P}<0.05$ vs. Con group. Western blotting, using GAPDH as a control, was conducted to determine autophagy-related protein (B) LC3 and (C) FoxO1 expression levels. CON, control; FoxO1, Forkhead box O1; LC3, microtubule-associated protein 1 light chain3.

$20 \mathrm{~min}$ in fresh 4\% paraformaldehyde solution (SigmaAldrich; Merck KGaA) and washed three times with PBS. Then, the cells were permeabilized using PBS containing $0.5 \%$ Triton-X 100 (Sigma-Aldrich; Merck KGaA) for $1 \mathrm{~h}$ and then blocked with PBS supplemented with $4 \%$ BSA and $0.3 \%$ Triton-X 100 for another $1 \mathrm{~h}$ at room temperature. Cells were incubated with anti-LC3 (1:100; Cell Signaling Technology) overnight at $4^{\circ} \mathrm{C}$. Cells were then washed three times with PBS and incubated with goat anti-rabbit and anti-mouse secondary antibodies for $1 \mathrm{~h}$ at room temperature. Digital images were obtained using an Olympus CKX41SF inverted microscope (magnification, x100; Olympus Corporation)

Transmission electron microscopy (TEM). INS-1 cells treated with $0.5 \mathrm{mmol} / 1 \mathrm{PA}$ and $10 \mu \mathrm{mol} / \mathrm{l} \mathrm{CQ}$ for $24 \mathrm{~h}$ at $37^{\circ} \mathrm{C}$ were harvested using a method described in our previous report (10). INS-1 cells were fixed with Karnovsky's fixative solution [1\% (v/v) paraformaldehyde, $2 \%$ (v/v) glutaraldehyde, $2 \mathrm{mmol} / 1$ calcium chloride and $100 \mathrm{mmol} / \mathrm{l}$ cacodylatebuffer (pH 7.4)] for $2 \mathrm{~h}$ at room temperature and washed with cacodylate buffer. After post-fixing with a fixative solution containing $1 \%$ (v/v) osmium tetroxide and $1.5 \%(\mathrm{v} / \mathrm{v})$ potassium ferrocyanide for $1 \mathrm{~h}$ at room temperature, the cells were dehydrated with $50-100 \%(\mathrm{v} / \mathrm{v})$ alcohol and stained with en bloc in $0.5 \%(\mathrm{v} / \mathrm{v})$ uranyl acetate at $4^{\circ} \mathrm{C}$ overnight. The cells were then embedded in Poly/Bed 812 resin (Pelco Scientific) and polymerized, after which they were sliced into $70 \mathrm{~nm}$ sections using a ReichertJung Ultracut E Ultramicrotome (Leica Microsystems GmbH) and stained with uranyl acetate and lead citrate at room temperature for $30 \mathrm{~min}$. The cells were observed and imaged under a TEM (EM902A; Carl Zeiss MicroImaging GmbH).

Statistical analysis. Data are presented as the mean \pm SD of $\geq 3$ independent experiments. Differences between the various groups were determined with by paired and unpaired Student's t-test using SPSS 19.0 software (IBM Corp.) or one-way ANOVA followed by Tukey's multiple comparison tests using GraphPad Prism Version 7 (GraphPad Software, Inc.). $\mathrm{P}<0.05$ was considered to indicate a statistically significant difference.

\section{Results}

Effects of PA in INS-1 cells injury, autophagy and FoxO1. INS-1 cells were incubated with various concentrations of PA
$(0,0.1,0.3$ and $0.5 \mathrm{mmol} / \mathrm{l})$ for $24 \mathrm{~h}$ to study the role of PA in the process of pancreatic $\beta$-cell injury, autophagy and FoxO1.

The viability of INS-1 cells was significantly decreased to $61 \%$ in the $0.5 \mathrm{mmol} / \mathrm{PA}$ group compared with the control group (Fig. 1A). These findings demonstrated that PA induces injury in INS-1 cells. PA at a concentration of $0.5 \mathrm{mmol} / \mathrm{l}$ significantly decreased the INS-1 cell viability, and thus this was selected as the suitable concentration for the PA group.

As LC3 is a landmark protein associated with autophagy, western blotting was used to measure the expression of LC3 in the INS-1 cells. The results demonstrated that the expression of LC3II/I was enhanced with the increasing concentrations of PA, compared with the control group (Fig. 1B). These results suggested that autophagy was activated in INS-1 cells in response to PA. The expression of FoxO1 was also analyzed via western blotting. The expression of FoxO1 was markedly decreased when INS-1 cells were cultured in $0.5 \mathrm{mmol} / 1 \mathrm{PA}$ compared with the control group (Fig. 1C). These results indicated that PA decreased the expression of FoxO1 in INS-1 cells.

Effect of autophagy on PA-induced INS-1 cell injury and FoxO1. INS-1 cells were treated with $0.5 \mathrm{mmol} / 1 \mathrm{PA}$ and $10 \mu \mathrm{mol} / \mathrm{l} \mathrm{CQ}$, an autophagy inhibitor, for $24 \mathrm{~h}$ to study the role of autophagy in PA-induced INS-1 cell injury and FoxO1.

Compared with the control group, the viability of INS-1 cell treated with CQ alone was significantly decreased (Fig. 2A). Moreover, the survival of INS-1 cells was significantly decreased in the PA + CQ group compared with the PA group (Fig. 2A). These results suggested that CQ accelerated PA-induce cytotoxicity, and that autophagy was essential for the survival of INS-1 cells, whether in a lipotoxic or a normal environment.

The expression of LC3II/Iwas increased in the PA+CQgroup compared with the PA group (Fig. 2B). Immunofluorescence was also used to detect LC3. The results identified that LC3 puncta were significantly increased in the PA group compared with in the control group (Fig. 2C). Moreover, the LC3 puncta numbers per viable cell was increased in the PA + CQ group compared with the PA group (Fig. 2C). TEM was used to examine the typical autophagic structures in INS-1 cells and provided evidence of autophagy activation. The double membrane autophagic vesicles containing cell organelles in the cytoplasm of INS-1 cells are integrated autophagosomes, shown via ultrastructural image analysis. It was found that the number of autophagic vacuoles was increased after PA 

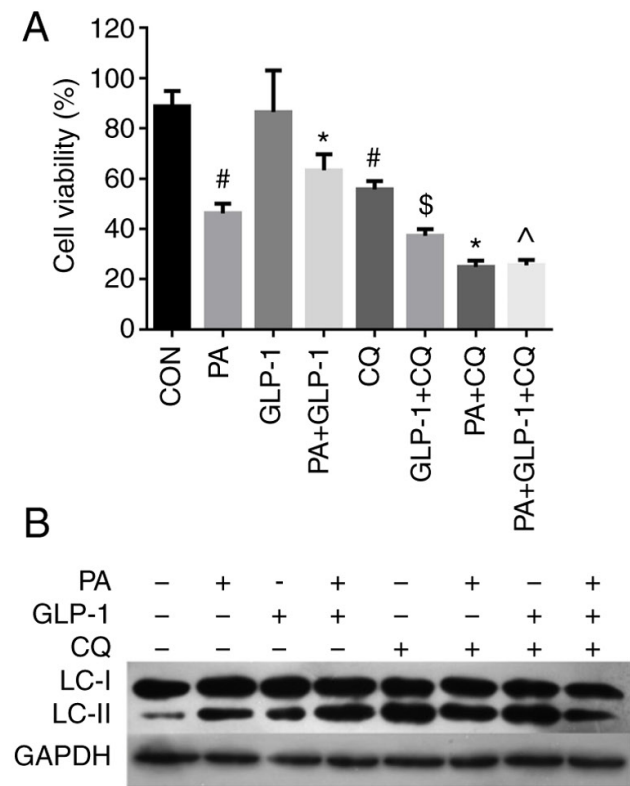

C

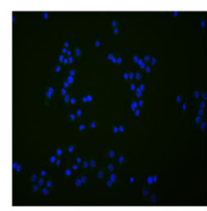
Control

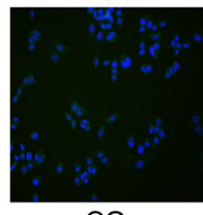

$\mathrm{CQ}$

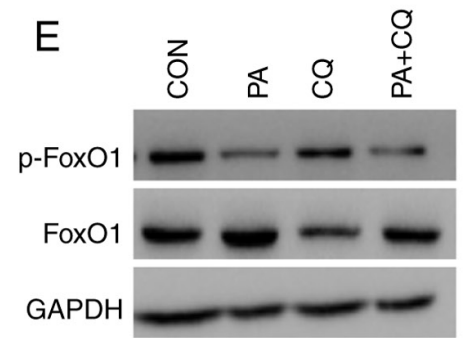

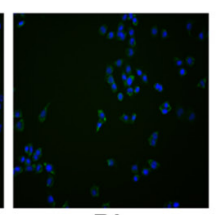

PA

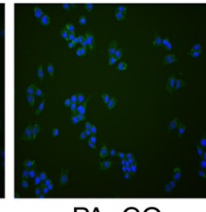

$\mathrm{PA}+\mathrm{CQ}$
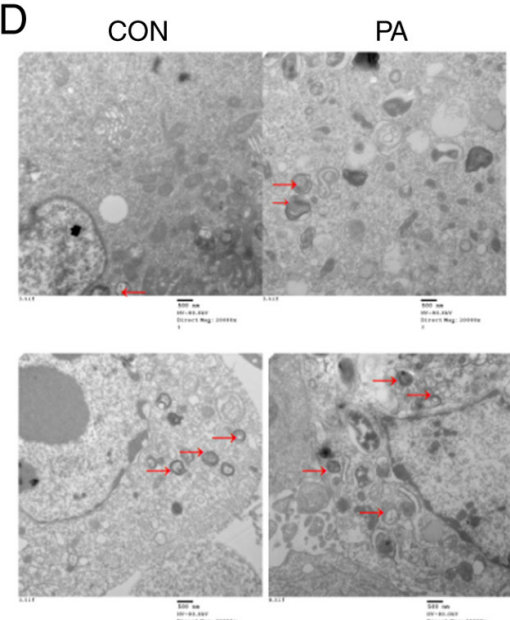

$\mathrm{CQ}$

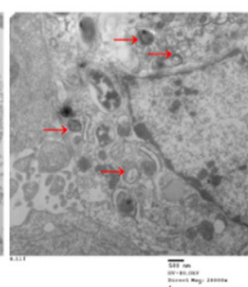

$\mathrm{PA}+\mathrm{CQ}$

Figure 2. Effect of autophagy in PA-induced INS-1 cells injury and FoxO1. (A) Cell Counting Kit-8 analysis of the viability of INS-1 cells in each group Data are presented as the mean $\pm \mathrm{SD}(\mathrm{n}=3) .{ }^{*} \mathrm{P}<0.05$ vs. $\mathrm{CON}$ group; ${ }^{*} \mathrm{P}<0.05$ vs. $\mathrm{PA}$ group; ${ }^{\$} \mathrm{P}<0.05$ vs. LIRA group; ${ }^{\wedge} \mathrm{P}<0.05$ vs. $\mathrm{PA}+\mathrm{LIRA}$ group. Data were analyzed by unpaired t-test. (B) Western blotting was used to detect the expression of LC3 in each group. (C) Immunofluorescence analysis of LC3 puncta in each group (magnification, x100). (D) Micromorphological changes in cellular organelles were examined via transmission electron microscopy. The red arrow points to autophagic vesicles (magnification, $x 20,000 ; n=3$ ). The red circles indicate the presence of autophagic vacuole structures. (E) Western blotting, using GAPDH as a control, was conducted to determine p-FoxO1 and FoxO1 protein expression levels in each group. FoxO1, Forkhead box O1; LC3, microtubuleassociated protein 1 light chain3; CON, control; PA, palmitate; CQ, chloroquine; GLP-1, glucagon-like peptide-1; p-, phosphorylated.

treatment (Fig. 2D). Compared with the PA group, there were numerous different forms of autophagosomes, which were enhanced when pre-treated with CQ (Fig. 2D). These results further suggested that autophagy was activated in INS-1 cells in response to PA. Furthermore, CQ aggravated the impairment of autophagy induced by PA in INS-1 cells. It was also identified that moderate autophagy had a protective effect in INS-1 cells and was necessary to maintain the normal architecture and function of INS-1 cells.

Next, it was investigated whether autophagy could regulate FoxO1 protein expression in INS-1 cells. As phosphorylation is critical for the FoxO1 function $(24,25)$, the levels of phosphorylated FoxO1 and total FoxO1 were detected in each group. There was no difference in the expression of p-FoxO1 between the PA group and the PA + CQ group (Fig. 2E). The results suggested that autophagy does not regulate the expression of FoxO1 in INS-1 cells.

Effects of liraglutide on PA-induced INS-1 cells injury, autophagy and FoxOl. To study the effects of liraglutide in PA-induced INS-1 cells injury, autophagy and FoxO1, INS-1 cells were cultured with $0.5 \mathrm{mmol} / \mathrm{l} \mathrm{PA}$ and $100 \mathrm{nmol} / \mathrm{l}$ liraglutide for $24 \mathrm{~h}$.

Treatment of INS-1 cells with PA decreased cell viability, which was partially prevented by co-treatment with liraglutide (Fig. 2A). To further identify the effects of the liraglutide in PA-induced INS-1 cells injury. Western blotting was used to detect the expression of cleaved caspase-3 in INS-1 cells. Treatment of INS-1 cells with PA increased the expression of cleaved caspase-3, which was partially prevented by co-treatment with liraglutide (Fig. 3A). However, there was no significant difference between the control and liraglutide group in terms of cell viability and apoptosis. These results suggested that liraglutide has a protective effect on PA-induced INS-1 cells rather than on normal conditions. Moreover, INS-1 cells viability was significantly decreased in PA + LIRA + CQ group compared with the PA + LIRA group (Fig. 2A). These results indicated that the activation of autophagy was a crucial component of liraglutide mediated PA-induced INS-1 cell survival, although other pathways affecting cell survival cannot be eliminated.

Western blotting was performed to investigate the effect of liraglutide on the expression of LC3 protein in INS-1 cells of each group. The expression of LC3II/I was increased in the LIRA group compared with the control group. Additionally, compared with the PA group, the expression of LC3II/I was increased in the PA + LIRA group, but was decreased when pretreated with CQ (Fig. 2B). These findings indicated that liraglutide partly reverses the effect of autophagy between CQ and PA-induced INS-1 cells. Immunofluorescence analysis of LC3 puncta was used to examine the effect of liraglutide in INS-1 cells. The green fluorescence intensity of LC3 was increased in the liraglutide group compared with the control group (Fig. 3B). These data demonstrated that liraglutide prevented INS-1 cells from PA-induced damage by enhancing the level of autophagy.

When compared with the control group, the phosphorylation of FoxO1 was decreased in the PA group (Fig. 3C). Pretreatment with liraglutide notably increased the PA-induced phosphorylation of FoxO1 in INS-1 cells (Fig. 3C). These 


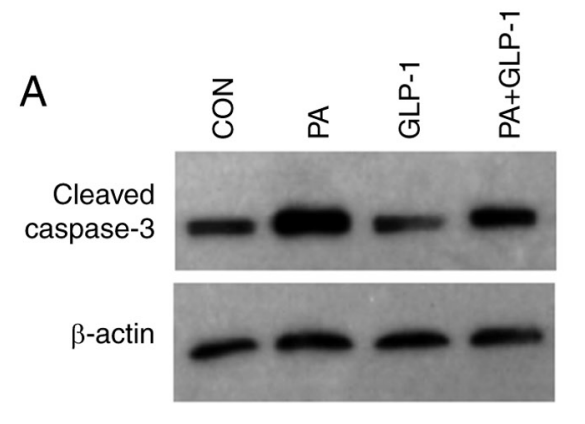

B
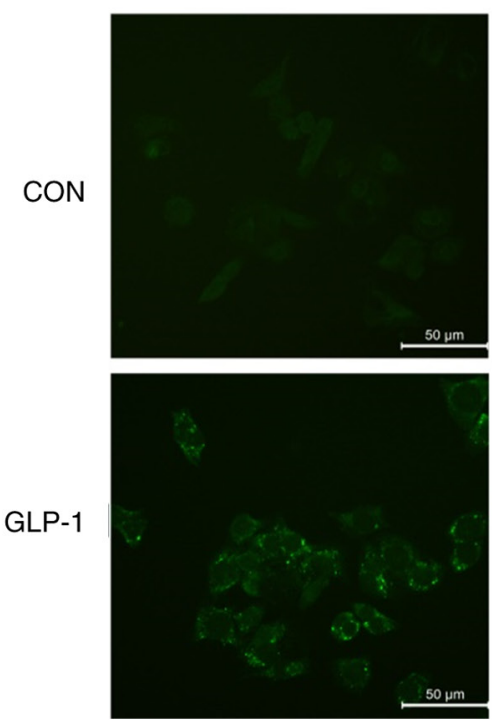
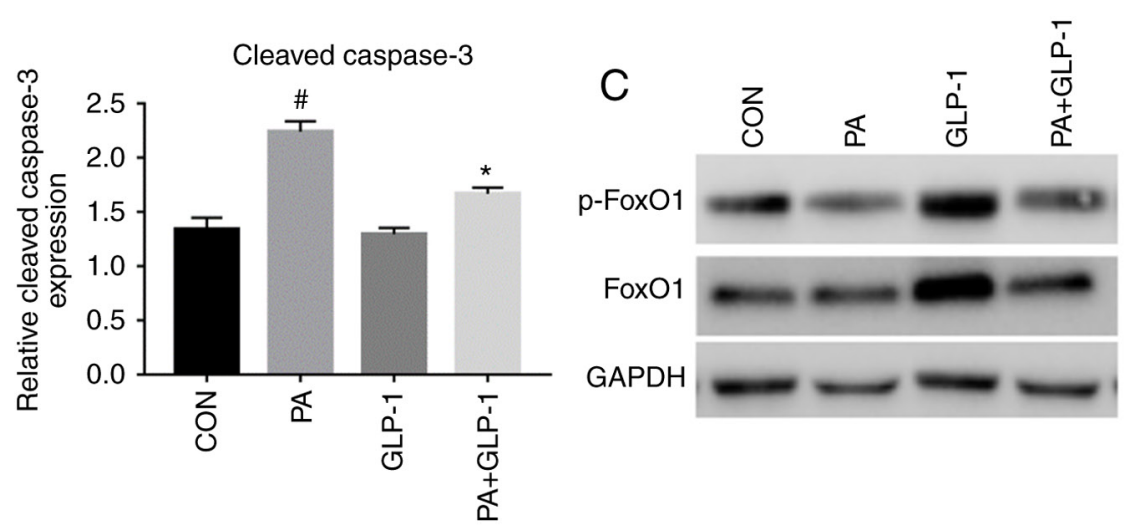

DAPI
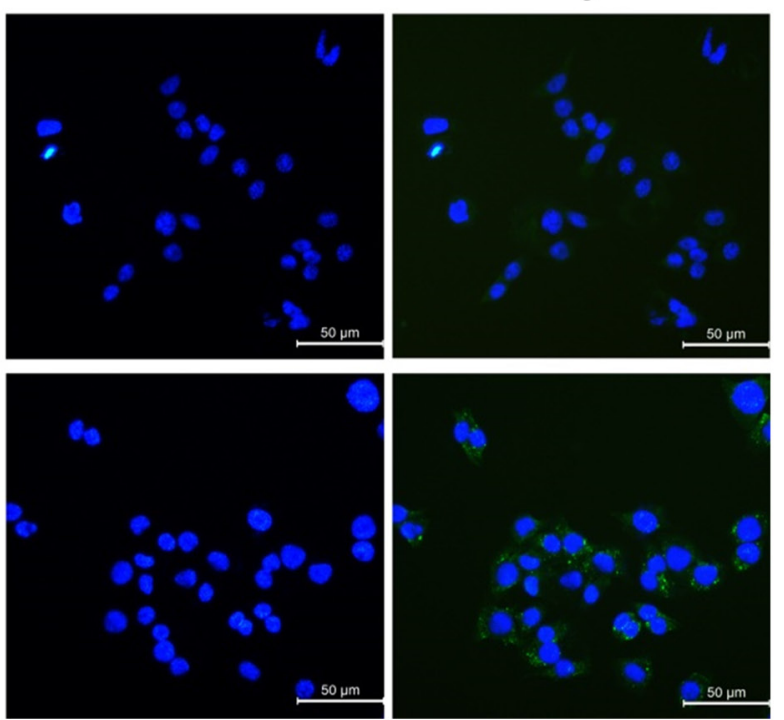

Figure 3. Effect of liraglutide in PA-induced INS-1 cells injury, autophagy and FoxO1. (A) Western blotting, using $\beta$-actin as a control, for determining cleaved caspase-3 protein expression. Data were analyzed by paired $\mathrm{t}$-test and are presented as the mean $\pm \mathrm{SD}(\mathrm{n}=3) .{ }^{\#} \mathrm{P}<0.05$ vs. CON group; ${ }^{*} \mathrm{P}<0.05$ vs. $\mathrm{PA}$ group. (B) Immunofluorescence analysis of LC3 puncta in control and GLP-1 group. Scale bar, $50 \mu \mathrm{m}$. (C). Western blotting was used to detect the expression levels of p-FoxO1 and FoxO1 in each group. FoxO1, Forkhead box O1; LC3, microtubule-associated protein 1 light chain3; CON, control; PA, palmitate; GLP-1, glucagon-like peptide-1; p-, phosphorylated.

results also indicated that liraglutide pretreatment could alleviate the reduction of $\mathrm{p}-\mathrm{FoxO} 1$ protein expression induced by PA in INS-1 cells.

Liraglutide, via FoxO1, improved the viability and autophagy induced by PA. To study the effects of FoxO1 in INS-1 cell injury, the present study interfered with the expression of FoxO1, and found that INS-1 cell viability was not different between the PA + siCON group and the PA + siFoxO1 group (Fig. 4A). The results demonstrated that interfering with the expression of FoxO1 did not regulate INS-1 cell viability in a high-fat environment. The viability of INS-1 cells was significantly increased in the LIRA + siCON group, compared with the PA + siCON group (Fig. 4A). However, INS-1 cell viability was substantially decreased in the LIRA + siFoxO1 group, compared with the LIRA + siCON group (Fig. 4A). These results indicated that silencing FoxO1 could attenuate the protection of liraglutide on the viability of INS-1 cells.

To confirm the role of FoxO1 in the effect of autophagy in INS-1 cells, the expression of FoxO1 was knocked down. FoxO1 expression was successfully suppressed in INS-1 cells that were transfected with siRNA targeting FoxO1 (Fig. 4B). The expression of LC3-II was decreased in the siFoxO1 group compared with the siCON group. The results demonstrated that silencing FoxO1 downregulated the level of autophagyassociated proteins in INS-1 cells (Fig. 4B). Collectively, the results suggested that FoxO1 restored autophagy.

\section{Discussion}

T2DM is characterized by insulin resistance and dysfunction of islet $\beta$-cells $(2,10)$. Substantial evidence indicates that oxidative stress, inflammatory response and ERs can lead to dysfunction of $\beta$-cells $(8,26,27)$. Functional damage and quantity reduction of islet $\beta$-cells serve a vital role in the occurrence and development of T2DM $(2,13)$. Moreover, hyperlipidemia is part of the main risk factors of diabetes (3). The toxic effect of FFA on $\beta$-cells is called lipotoxicity. Islet $\beta$-cell apoptosis induced by FFA is currently considered to be one of the main pathogenesis of T2DM, but its exact mechanism remains unknown. PA is a FFA that is recognized as the most widely used molecule to damage islet $\beta$-cells via lipotoxicity (21). Therefore, the present study used PA to treat INS-1 cells to 

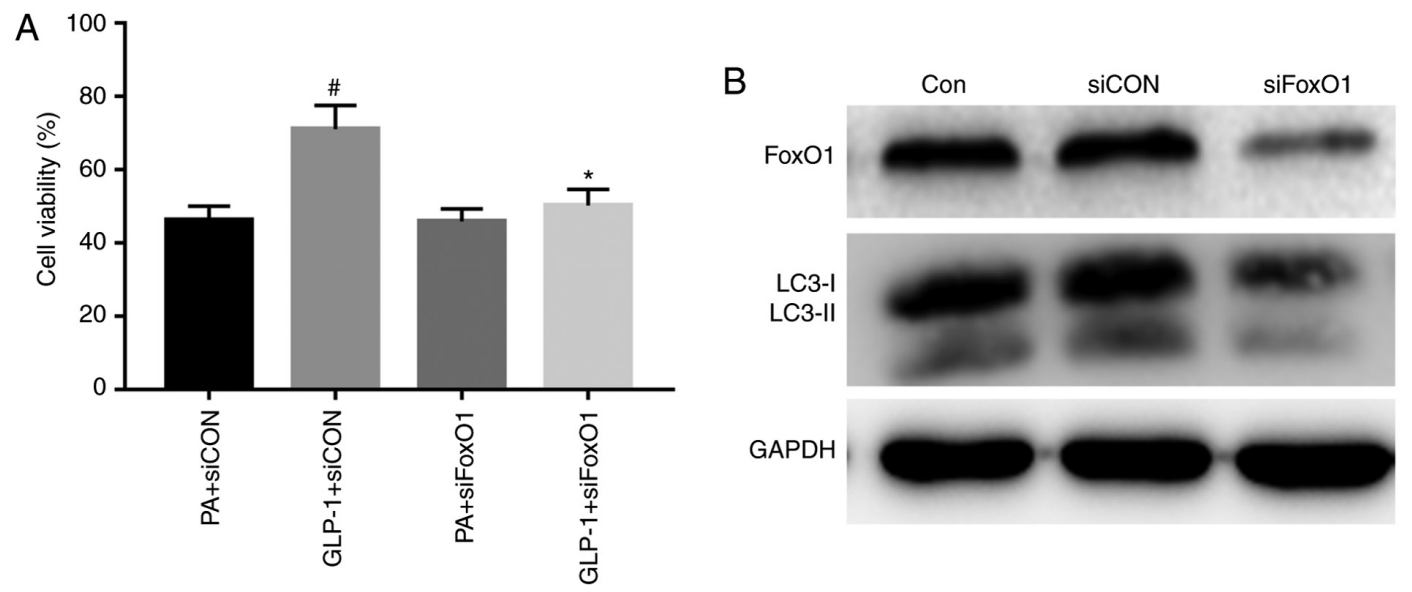

Figure 4. Liraglutide improves the viability and autophagy induced by PA via FoxO1. (A) A Cell Counting Kit-8 assay was used to analyze the viability of INS-1 cells in each group. Data were analyzed by paired t-test and are presented as the mean $\pm \mathrm{SD}(\mathrm{n}=3)$. "P<0.05 vs. GLP-1 + siCON group; ${ }^{*} \mathrm{P}<0.05$ vs. PA + siCON group. (B) Western blotting was used to detect the expression levels of FoxO1 and LC3 in each group. FoxO1, Forkhead box O1; LC3, microtubuleassociated protein 1 light chain3; CON, control; PA, palmitate; GLP-1, glucagon-like peptide-1; si, small interfering RNA.

establish a pancreatic $\beta$-cells injury model, which caused in vitro $\beta$-cell dysfunction in T2DM.

In the present experiment, INS-1 cells were treated with different concentrations of PA. The results demonstrate that INS-1 cell viability was decreased with the increasing concentrations of PA. Our previous study reported similar results that PA significantly reduced islet $\beta$-cell viability in a dosedependent manner (28). The present study incubated INS-1 cells with $0.5 \mathrm{mmol} / \mathrm{l} \mathrm{PA}$ for $24 \mathrm{~h}$ and found that the expression of cleaved caspase-3 was increased. Moreover, it was identified that chronic exposure to PA increased INS-1 cells injury. These results are in line with the previous findings and provide a possible mechanism to link lipotoxicity to T2DM (29).

Autophagy regulates the degradation of organelles and proteins in eukaryotic cells, which helps to maintain cellular homeostasis (6). As autophagy serves a critical role in maintaining cell homeostasis, autophagy is indispensable in $\beta$-cells physiology (7). A lack of autophagy promotes the impairment of islet $\beta$-cells function and mass, eventually lead to the onset of diabetes $(1,10,12)$. The present findings demonstrated that the autophagy inhibitor CQ further decreased the survival rate of INS-1 cells incubated in PA. The results also indicated that autophagy promoted pancreatic $\beta$-cells survival, which was consistent with our previous studies $(1,28)$. It was found that autophagy protected $\beta$-cells from PA-induced injury and exerted a protective role in the occurrence of T2DM. Autophagy also serves a major role in maintaining islet $\beta$-cells function and survival. To further investigate the interaction between autophagy and lipotoxicity in INS-1 cells, the present study cultured INS-1 cells in a lipotoxic environment and found that the autophagy hallmark protein LC3-II was enhanced with increasing PA concentrations. These results were further confirmed by immunofluorescence results, which identified that LC3 puncta were significantly increased with $0.5 \mathrm{mmol} / \mathrm{l}$ PA. The current immunofluorescence results were consistent with the results of TEM analysis. The present data are consistent with the prior studies showing that elevated serum levels of FFA mediated induction of $\beta$-cell autophagy $(28,30)$.

The increase of autophagosome formation and the decrease of lysosomal fusion and degradation can result in the accumulation of LC3-II (6,7). To further verify whether LC3-II accumulation induced by PA resulted from impaired clearance due to defective fusion with lysosomes or from the true autophagic flux, the present study measured LC3-II in the presence of CQ and found that the level of LC3-II protein was increased. The results were also confirmed via immunofluorescence, in which the number of LC3 puncta increased. In addition, TEM identified that the number of autophagic vacuoles was increased in the CQ group and PA + CQ group. The present data are in agreement with the previous studies $(5,7,28)$, which reported that CQ increased PA-induced defective autophagic turnover in INS-1 cells.

Liraglutide, a GLP-1 analog, serves a pivotal role in pancreatic $\beta$-cell proliferation, anti-apoptotic function and autophagy $(1,3,4,21)$. Liraglutide protects islet $\beta$-cells from FFA and affects glucolipid metabolism by activating autophagy (3). While it is known that GLP-1 regulates $\beta$-cell autophagy by defending against oxidative stress and ERs (31), further studies are required to elucidate the mechanisms between liraglutide and autophagy in pancreatic $\beta$-cells. The present study investigated the protective effect of liraglutide on PA-induced INS-1 cells injury. Liraglutide significantly inhibited the expression of cleaved caspase-3 protein in INS-1 cells induced by PA acid, as well as increased the survival and autophagy of INS-1 cells induced by PA. This was verified by an increase in LC3-II formation and cell viability. However, this effect can be reversed by the autophagy inhibitor CQ. These data demonstrated that liraglutide exerted its cytoprotective effects on PA-induced INS-1 cells injury by regulating autophagy, and these are similar to a previous study (28).

It has been revealed that the GLP-1 receptor agonist exendin- 4 improves the $\beta$-cell survival induced by glucolipotoxicity and the effect was dependent on the restoration of autophagic signaling (31). GLP-1 can change the subcellular distribution of FoxO1 and promote its translocation in pancreatic $\beta$-cells (32). The GLP-1 receptor inhibitor exendin9-39 downregulates the expression of p-FoxO1 in NIT-1 insulinoma cells (33). Moreover, the GLP-1 receptor agonist exendin-4 upregulates adiponectin levels in adipocytes via FoxO1, both in vitro and in vivo (34). Shao et al (21) reported that liraglutide markedly improved 


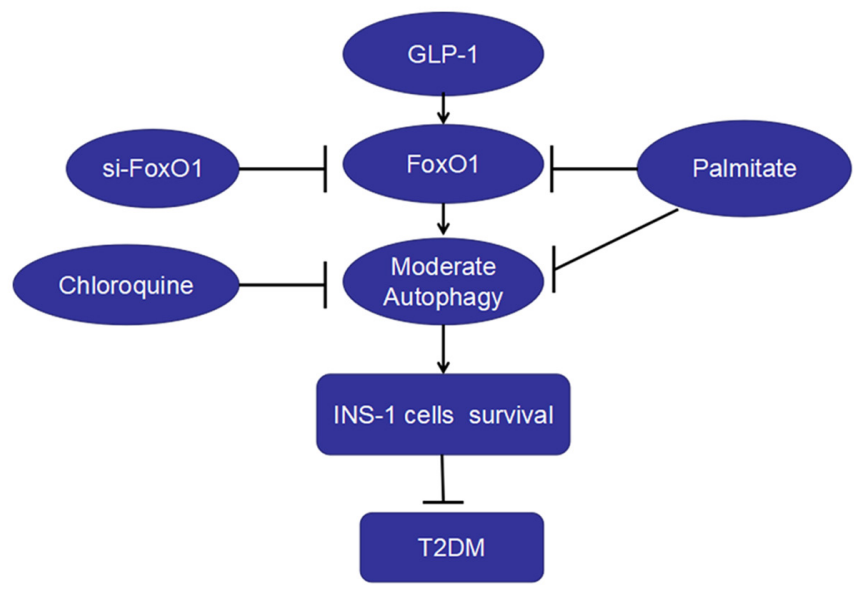

Figure 5. GLP-1 analog liraglutide induces autophagy and improves INS-1 cell survival in palmitate medium by activating FoxO1. Palmitate downregulated FoxO1, caused the dysfunction of autophagy and decreased INS-1 cell survival, thus inducing DM. The autophagy inhibitor chloroquine decreased the viability of INS-1 cells. Moreover, siFoxO1 suppressed autophagy and decreased the protection of liraglutide on the viability of INS-1 cells. Thus, liraglutide protected islet $\beta$-cells from T2DM. FoxO1, Forkhead box O1; LC3, microtubule-associated protein 1 light chain3; GLP-1, glucagon-like peptide-1; siRNA, small interfering RNA; T2DM, type 2 diabetes mellitus.

$\beta$-cells function under FFA, and the protective action of liraglutide was mediated by the PI3K/AKT/FoxO1 pathway. Exendin can also increase the levels of $\mathrm{p}-\mathrm{FoxO} 1$ via protein kinase A in $\beta$-cells (22). Geniposide enhances the phosphorylation of Foxol and inhibits apoptosis in PA-treated INS-1 cells by activating the GLP-1 receptor (35). Furthermore, GLP-1 enhances FoxO1 acetylation and stimulate $\beta$-cells mass expansion (36). Therefore, the interactions between liraglutide and FoxO1 autophagy are worth investigating in pancreatic $\beta$-cells. However, whether liraglutide mediates $\beta$-cell autophagy via FoxO1 remains unknown. The present study demonstrated that liraglutide increased the expression of p-FoxO1 protein in PA-induced INS-1 cells. Furthermore, FoxO1 silencing attenuated the protective effect of liraglutide on INS-1 cells. Collectively, the results indicated that liraglutide protected INS-1 cells against PA by upregulating the expression of FoxO1.

To determine the effect of FoxO1-mediated cell viability and autophagy in INS-1 cells, the present study blocked the expression of FoxO1 in INS-1 cells. FoxO1 is a downstream effector of insulin/insulin-like growth factor-I signaling and can be regulated by Akt via phosphorylation, following nuclear exclusion $(13,24)$. Furthermore, FoxO1 is a key regulatory factor governing a variety of physiological functions, including the pancreatic $\beta$-cells proliferation, apoptosis and autophagy $(14,15,18,37)$. FoxO1 also protects pancreatic $\beta$-cells by inhibiting thioredoxin-interacting protein transcription (38). In the present study, the expression of FoxO1 was downregulated with the increase in concentrations of PA. FoxO1 silencing suppressed the viability of liraglutide-pretreated INS-1 cells. These data suggested that FoxO1 silencing attenuated the protective effect of liraglutide on INS-1 cells. The relationship between FoxO1 and autophagy was examined, and the present results suggested that blocking the FoxO1 expression suppressed the autophagy-related protein LC3-II in INS-1 cells. Liu et al (39) revealed that FoxO1 antagonist treatment induces similar responses in adipocytes. However, in the present study, the autophagy inhibitor CQ did not affect the expression of p-FoxO1 in INS-1 cells. The current results were consistent with previously reported findings that the effect of autophagy can be regulated by FoxO1 but the autophagy inhibitor 3-methyadenine had no effect on FoxO1 in vascular endothelial cells (19). These results indicated that FoxO1 improved the viability of INS-1 cells against PA by upregulating the expression of autophagy-associated proteins. However, the mechanism of FoxO1 regulating autophagy requires further investigation.

In the present study, it was found that: i) PA decreased the viability, increased apoptosis and autophagy and depressed the expression of FoxO1 in INS-1 cells; ii) inhibiting the effect of autophagy decreased the survival and the expression of FoxO1 in INS-1 cells; iii) liraglutide increased INS-1 cell survival, autophagy and the expressions of FoxO1 induced by PA; and iv) FoxO1 silencing decreased the level of autophagy and the protection of liraglutide on the viability of INS-1 cells. Therefore, liraglutide protected INS-1 cells against PA-induced injury, which may occur via the upregulation of autophagy mediated by FoxO1 (Fig. 5).

There are certain limitations to the present study. First, PA was used to test the cell viability, apoptosis and autophagy of pancreatic $\beta$-cells, based on its lipotoxicity $(40,41)$. Previous studies also proposed that PA could participate in apoptosis via reactive oxygen species and ERs generation in isolated rat islets of Langerhans $(28,29,37,42)$. Therefore, further studies are required obtain an in-deep insight into the role of PA in apoptosis. Second, the main purpose of the present study was to examine the mechanism of liraglutide on the autophagy of islet $\beta$-cells; however, the improvement of insulin secretion function in $\beta$-cells due to the autophagy effect of liraglutide was not been studied. Third, GLP-1 regulates autophagy in multiple signal pathways, including PI3K/Akt and 5' AMP-activated protein kinase (43). Therefore, further research to investigate the pathway between GLP-1 and autophagy is required. Moreover, there are some limitations with regards to the model. For instance, CQ does not simply prevent autophagy, but blocks the pathway leading to a build-up of autophagosomes and lysosomes. Therefore, dysfunctional autophagy may have a much larger impact on cell survival and function than simply preventing autophagy. In addition, due to the limited funds, intracellular phosphorylation of FoxO1/total FoxO1 was not examined in the present study. However, a literature search was conducted and it was found that the effects of FoxO1 depend on its activation, which can be influenced by its abundance, post-transcriptional modification, nuclearcytoplasmic shuttling and subcellular localization $(13,17,18)$. Among these activating factors, phosphorylation is critical for the FoxO1 function $(24,25)$. Finally, the present study lacked in vivo experiments, which will further validate the use of liraglutide for diabetes treatment.

In conclusion, to the best of our knowledge, this is the first study that demonstrated that the GLP-1 agonist liraglutide protected INS-1 cells against PA-induced injury via the upregulation of autophagy mediated by FoxO1. This may provide a novel theoretical basis and experimental support for liraglutide in the treatment of diabetes. 


\section{Acknowledgements}

Not applicable.

\section{Funding}

The present study was supported by the National Natural Science Foundation of China (grant no. 81770820).

\section{Availability of data and materials}

All data generated or analyzed during the present study are included in this published article.

\section{Authors' contributions}

XDL performed the cell experiments. XDL and SSH performed CCK8 and immunofluorescence assays and western blot analysis, XDL and TTW performed transmission electron microscopy. XDL and YBL contributed to the study design and writing of the manuscript. All authors read and approved the final manuscript.

\section{Ethics approval and consent to participate}

Not applicable.

\section{Patient consent for publication}

Not applicable.

\section{Competing interests}

The authors declare that they have no competing interests.

\section{References}

1. Chen ZF, Li YB, Han JY, Yin JJ, Wang Y, Zhu LB and Xie GY: Liraglutide prevents high glucose level induced insulinoma cells apoptosis by targeting autophagy. Chin Med J (Engl) 126: 937-941, 2013.

2. Chen ZF, Li YB, Han JY, Wang J, Yin JJ, Li JB and Tian H: The double-edged effect of autophagy in pancreatic beta cells and diabetes. Autophagy 7: 12-16, 2011.

3. Wang J, Wu J, Wu H, Liu X, Chen Y, Wu J, Hu C and Zou D: Liraglutide protects pancreatic $\beta$-cells against free fatty acids in vitro and affects glucolipid metabolism in apolipoprotein E-/- mice by activating autophagy. Mol Med Rep 12: 4210-4218, 2015.

4. Fan M, Jiang H, Zhang Y, Ma Y, Li L and Wu J: Liraglutide enhances autophagy and promotes pancreatic $\beta$ cell proliferation to ameliorate type 2 diabetes in high-fat-fed and streptozotocintreated mice. Med Sci Monit 24: 2310-2316, 2018

5. Li Q, Jia S, Xu L, Li B and Chen N: Metformin-induced autophagy and irisin improves INS-1 cell function and survival in high-glucose environment via AMPK/SIRT1/PGC-1 $\alpha$ signal pathway. Food Sci Nutr 7: 1695-1703, 2019.

6. Lee YH, Kim J, Park K and Lee MS: $\beta$-cell autophagy: Mechanism and role in $\beta$-cell dysfunction. Mol Metab 27S: S92-S103, 2019.

7. Toledo M and Singh R: Complement C3 and autophagy keep the $\beta$ cell alive. Cell Metab 29: 4-6, 2019.

8. Liu H, Yin JJ, Cao MM, Liu GD, Su Y and Li YB: Endoplasmic reticulum stress induced by lipopolysaccharide is involved in the association between inflammation and autophagy in INS-1 cells Mol Med Rep 16: 5787-5792, 2017.
9. King BC, Kulak K, Krus U, Rosberg R, Golec E, Wozniak K, Gomez MF, Zhang E, O'Connell DJ, Renström E, et al: Complement component $\mathrm{C} 3$ is highly expressed in human pancreatic islets and prevents $\beta$ cell death via ATG16L1 interaction and autophagy regulation. Cell Metab 29: 202-210.e6, 2019.

10. Zhu L-B, Cao M-M, Wang J, Su Y, Jiang W, Liu GD and Li YB: Role of autophagy in LPS-induced inflammation in INS-1 cells. Mol Med Rep 19: 5211-5218, 2019.

11. Zhou X-T, Pu Z-J, Liu L-X, Li GP, Feng JL, Zhu HC and Wu LF: Inhibition of autophagy enhances adenosine-induced apoptosis in human hepatoblastoma HepG2 cells. Oncol Rep 41: 829-838, 2019.

12. Quan W, Hur KY, Lim Y, Oh SH, Lee JC, Kim KH, Kim GH, Kim SW, Kim HL, Lee MK, et al: Autophagy deficiency in beta cells leads to compromised unfolded protein response and progression from obesity to diabetes in mice. Diabetologia 55: 392-403, 2012

13. Kitamura T: The role of FOXO1 in $\beta$-cell failure and type 2 diabetes mellitus. Nat Rev Endocrinol 9: 615-623, 2013.

14. Zhang T, Kim DH, Xiao X,LeeS, Gong Z, Muzumdar R, CalabuigNavarro V, Yamauchi J, Harashima H, Wang R, et al: FoxO1 Plays an Important Role in Regulating $\beta$-Cell Compensation for Insulin Resistance in Male Mice. Endocrinology 157: 1055-1070, 2016.

15. He W, Zhang A, Qi L, Na C, Jiang R, Fan Z and Chen J: FOXO1, a potential therapeutic target, regulates Autophagic flux, oxidative stress, mitochondrial dysfunction, and apoptosis in human cholangiocarcinoma QBC939 cells. Cell Physiol Biochem 45: 1506-1514, 2018

16. Mo X, Wang X, Ge Q and Bian F: The effects of SIRT1/FoxO1 on LPS induced INS-1 cells dysfunction. Stress 22: 70-82, 2019.

17. Kitamura YI, Kitamura T, Kruse JP, Raum JC, Stein R, Gu W and Accili D: FoxO1 protects against pancreatic beta cell failure through NeuroD and MafA induction. Cell Metab 2: 153-163, 2005.

18. Kobayashi M,KikuchiO,Sasaki T, Kim HJ, Yokota-Hashimoto H, Lee YS, Amano K, Kitazumi T, Susanti VY, Kitamura YI, et al: FoxO1 as a double-edged sword in the pancreas: Analysis of pancreas- and $\beta$-cell-specific FoxO1 knockout mice. Am J Physiol Endocrinol Metab 302: E603-E613, 2012.

19. Wu Q, Hu Y, Jiang M, Wang F and Gong G: Effect of autophagy regulated by Sirt1/FoxO1 pathway on the release of factors promoting thrombosis from vascular endothelial cells. Int J Mol Sci 20: 4132, 2019.

20. Ren H, Shao Y, Wu C, Ma X, Lv C and Wang Q: Metformin alleviates oxidative stress and enhances autophagy in diabetic kidney disease via AMPK/SIRT1-FoxO1 pathway. Mol Cell Endocrinol 500: 110628, 2020.

21. Shao S, Nie M, Chen C, Chen X, Zhang M, Yuan G, Yu X and Yang Y: Protective action of liraglutide in beta cells under lipotoxic stress via PI3K/Akt/FoxO1 pathway. J Cell Biochem 115: 1166-1175, 2014.

22. Muhammad AB, Xing B, Liu C, Naji A, Ma X, Simmons RA and Hua X: Menin and PRMT5 suppress GLP1 receptor transcript and PKA-mediated phosphorylation of FOXO1 and CREB. Am J Physiol Endocrinol Metab 313: E148-E166, 2017.

23. Yin J, Wang Y, Gu L, Fan N, Ma Y and Peng Y: Palmitate induces endoplasmic reticulum stress and autophagy in mature adipocytes: Implications for apoptosis and inflammation. Int J Mol Med 35: 932-940, 2015.

24. Xing YQ, Li A, Yang Y, Li XX, Zhang LN and Guo HC: The regulation of FOXO1 and its role in disease progression. Life Sci 193: 124-131, 2018.

25. Chen B, Zhou W, Zhao W, Yuan P, Tang C, Wang G, Leng J, Ma J, Wang X, Hui Y, et al: Oxaliplatin reverses the GLP-1Rmediated promotion of intrahepatic cholangiocarcinoma by altering FoxO1 signaling. Oncol Lett 18: 1989-1998, 2019.

26. Wang F, Yin J, Ma Y, Jiang H and Li Y: Vitexin alleviates lipopolysaccharide-induced islet cell injury by inhibiting HMGB1 release. Mol Med Rep 15: 1079-1086, 2017.

27. Cao MM, Lu X, Liu GD, Su Y, Li YB and Zhou J: Resveratrol attenuates type 2 diabetes mellitus by mediating mitochondrial biogenesis and lipid metabolism via Sirtuin type 1. Exp Ther Med 15: 576-584, 2018.

28. Fu J, Nchambi KM, Wu H, Luo X, An X and Liu D: Liraglutide protects pancreatic $\beta$ cells from endoplasmic reticulum stress by upregulating MANF to promote autophagy turnover. Life Sci 252: 117648, 2020. 
29. Bugliani M, Mossuto S, Grano F, Suleiman M, Marselli L, Boggi U, De Simone P, Eizirik DL, Cnop M, Marchetti P, et al: Modulation of autophagy influences the function and survival of human pancreatic beta cells under endoplasmic reticulum stress conditions and in type 2 diabetes. Front Endocrinol (Lausanne) 10 52, 2019.

30. Chu KY, O'Reilly L, Mellet N, Meikle PJ, Bartley C and Biden TJ: Oleate disrupts cAMP signaling, contributing to potent stimulation of pancreatic $\beta$-cell autophagy. J Biol Chem 294: $1218-1229,2019$

31. Zummo FP, Cullen KS, Honkanen-Scott M, Shaw JAM, Lovat PE and Arden C: Glucagon-Like peptide 1 protects pancreatic $\beta$-cells from death by increasing autophagic flux and restoring lysosomal function. Diabetes 66: 1272-1285, 2017.

32. Zhang J, Tokui Y, Yamagata K, Kozawa J, Sayama K, Iwahashi H, Okita K, Miuchi M, Konya H, Hamaguchi T, et al: Continuous stimulation of human glucagon-like peptide-1 (7-36) amide in a mouse model (NOD) delays onset of autoimmune type 1 diabetes. Diabetologia 50: 1900-1909, 2007.

33. Wu Y-J, Wu Y-B, Fang Z-H, Chen MQ, Wang YF, Wu CY and Lv MA: Danzhi Jiangtang capsule mediates NIT-1 insulinoma cell proliferation and apoptosis by GLP-1/Akt signaling pathway. Evid Based Complement Alternat Med 2019: 5356825, 2019.

34. Wang A, Li T, An P, Yan W, Zheng H, Wang B and Mu Y: Exendin-4 upregulates adiponectin level in adipocytes via Sirt1/ Foxo-1 signaling pathway. PLoS One 12: e0169469, 2017.

35. Liu J, Yin F, Xiao H, Guo L and Gao X: Glucagon-like peptide 1 receptor plays an essential role in geniposide attenuating lipotoxicity-induced $\beta$-cell apoptosis. Toxicol In Vitro 26 : 1093-1097, 2012.

36. Bastien-Dionne PO, Valenti L, Kon N, Gu W and Buteau J: Glucagon-like peptide 1 inhibits the sirtuin deacetylase SirT1 to stimulate pancreatic $\beta$-cell mass expansion. Diabetes 60 : $3217-3222,2011$
37. Shaklai S, Grafi-Cohen M, Sharon O, Sagiv N, Shefer G, Somjen D and Stern N: Pancreatic beta-cell proliferation induced by estradiol-17 $\beta$ is Foxol dependent. Horm Metab Res 50: 485-490, 2018

38. Kibbe C, Chen J, Xu G, Jing G and Shalev A: FOXO1 competes with carbohydrate response element-binding protein (ChREBP) and inhibits thioredoxin-interacting protein (TXNIP) transcription in pancreatic beta cells. J Biol Chem 288: 23194-23202, 2013.

39. Liu L, Zheng LD, Zou P, Brooke J, Smith C, Long YC, Almeida FA, Liu D and Cheng Z: FoxO1 antagonist suppresses autophagy and lipid droplet growth in adipocytes. Cell Cycle 15: 2033-2041, 2016

40. Min S, Zhu T, Tong J, Caidan R, Wang K, Kai G, Zhang W, $\mathrm{Ru} \mathrm{L}$, Pengcuo J and Tong L: Screening active components from Rubus amabilis for pancreatic $\beta$-cells protection. Pharm Biol 58: 674-685, 2020

41. Liu X, Zeng X, Chen X, Luo R, Li L, Wang C, Liu J, Cheng J, $\mathrm{Lu}$ Y and Chen Y: Oleic acid protects insulin-secreting INS-1E cells against palmitic acid-induced lipotoxicity along with an amelioration of ER stress. Endocrine 64: 512-524, 2019.

42. Liu C, Fu Y,Li CE, Chen T and Li X: Phycocyanin-functionalized selenium nanoparticles reverse palmitic acid-induced pancreatic $\beta$ cell apoptosis by enhancing cellular uptake and blocking reactive oxygen species (ROS)-mediated mitochondria dysfunction. J Agric Food Chem 65: 4405-4413, 2017.

43. Yang Y, Fang H, Xu G, Zhen Y, Zhang Y, Tian J, Zhang D, Zhang $\mathrm{G}$ and $\mathrm{Xu}$ J: Liraglutide improves cognitive impairment via the AMPK and PI3K/Akt signaling pathways in type 2 diabetic rats. Mol Med Rep 18: 2449-2457, 2018.

This work is licensed under a Creative Commons Attribution-NonCommercial-NoDerivatives 4.0 International (CC BY-NC-ND 4.0) License. 